\title{
Interaction between a falling sphere and the structure of a non-Newtonian yield-stress fluid
}

\author{
Nicolò R. Sgreva ${ }^{\mathrm{a}, *}$, Anne Davaille ${ }^{\mathrm{a}}$, Ichiro Kumagai ${ }^{\mathrm{b}}$, Kei Kurita ${ }^{\mathrm{c}}$ \\ ${ }^{a}$ Université Paris-Saclay, CNRS, FAST, 91405, Orsay, France. \\ ${ }^{b}$ School of Science and Engineering, Meisei University, Hino, Tokyo 191-8506, Japan \\ ${ }^{c}$ Earthquake Research Institute, University of Tokyo, Tokyo, Japan
}

\begin{abstract}
We present an experimental study using mixtures of aqueous superabsorbent polymers (SAP) where we systematically investigate the influence of the size of grains that make up the fluid structure on the effective rheology and its domain of validity. In water, these polymer powder grains swell up to 200 times and form gel grains whose size can be controlled by controlling the size of the initial powder. The rheology of this mixture (water and touching grains) combines viscous, elastic and plastic aspects and can be characterized using the free-fall of spheres of different diameters (between 3 and $25 \mathrm{~mm}$ diameter) and densities (from 2200 to $15000 \mathrm{~kg} / \mathrm{m}^{3}$ ). As the typical size of the gel grains was varied between 1 and $8 \mathrm{~mm}$, there is a range where it becomes comparable to the size of the falling spheres. We observe five different motion regimes. (1) A linear regime where the sphere has a rapid and linear fall and reaches a constant terminal velocity. (2) An irregular regime where the sphere's velocity varies around a constant value. (3) An intermittent regime where periods of no-motion and periods of irregular falls follow one another.
\end{abstract}

*nicolo.sgreva@u-psud.fr 
(4) A slow fall regime where the sphere's speed progressively decreases in a logarithmic way. And (5) a no-motion regime when spheres are not heavy enough to overcome the yield stress of the mixture, or are too small compared to the grain size. Besides these five regimes, we find that the effective yield stress and effective consistency of the mixtures always increase with the grain size, suggesting that the effective rheology depends on the size of the grains which constitute the fluid structure. Moreover, the critical Yield number $\left(Y_{c}\right)$ above which there is no motion decreases as the sphere to grain diameters ratio becomes smaller than 2 . This shows the role of the fluid structure also on the critical condition of motion under which the sphere is trapped.

Keywords: Yield stress, Falling sphere, SAP, Irregular motion, Intermittent motion

\section{Introduction}

Yield-stress fluids are a broad category of fluids which have been inten3 sively studied in the last decades due to their key roles both in industry (e.g. 4 drilling fluids, cement pastes, foams) and in natural phenomena (e.g. lava 5 and mud flows, avalanches, landslides). Very often, the material consists 6 typically of a suspension of particles in a liquid. When the particle volume 7 fraction increases, they become in close contact with one another, and the stresses without flowing. A so-called yield-stress fluid does not flow if the applied stresses are not high enough to unjam the structure, but it does flow when the stresses become larger than a threshold value, the yield stress $\left(\sigma_{Y}\right)$. The latter introduces a non-linearity into the material rheology, which 
strongly impacts on its dynamics $[1,2,3,4]$. An example of these materials are soft particle glasses. Above the yield stress, their flow results from the concurrence of particles elastic deformations and plastic events during which particles rearrange [5]. Plastic local events can influence each other and lead to non-local elastic relaxation. This collective rearrangement can be the reason of spatial variation of viscosity in an homogeneous stress field in emulsions $[6,7]$. Other finite size effects to the bulk rheology of a jammed system appear due to concentration gradients of particles in non-Brownian particle suspensions [8] or due to structural heterogeneities in polymers [9].

Several rheological models have been proposed to describe the rheology above the yield stress, such as the Bingham and the Herschel-Bulkley "HB" models. One issue is the domain of validity of these rheology models: how well do they describe the fluid behavior close to the yield stress and how much larger than the microstructure typical length scale must be the flow length scale? For example, if we take the simple (and well known in Newtonian fluids) problem of a solid sphere settling in a particulate fluid, when and how will the fluid microstructure interact with the sphere motion? There are two end-members depending on the size ratio between the fluid microstructure $d_{g}$ (e.g. the size of the particles in suspension in the liquid) and the falling sphere $d_{s}$. When $d_{g} / d_{s}>>1$, the sphere will fall through a porous medium, moving along the liquid channels between particles, and when $d_{g} / d_{s}<<1$, the sphere will see a continuum with an effective rheology (e.g. "HB" model). But what happens for intermediate $d_{g} / d_{s}$ ?

The $d_{g} / d_{s}<<1$ case has been well studied for viscoplastic fluids, such as Carbopol [2]: the sphere reaches a steady-state downward motion only 
if a critical force is overcome, otherwise the fall is prevented [10, 11, 12]. However, the accurate value of this critical force is still debated and it varies, for example, considering or not the elasticity of the material [13]. Once the sphere is set in motion, crucial aspects are the determination of the drag coefficient $[10,11,14,15]$ and the determination of the shape and position of the yield surface that separates the solid-like region from the fluid-like one. The yield surface and the drag force on the sphere have first been determined numerically and analytically for a Bingham fluid by Beris et al. [10] and subsequently for an HB fluid numerically [14, 16, 15] and experimentally by Tabuteau et al. [11] in Carbopol. Within the liquid-like region, the flow in Carbopol is confined nearby the sphere, within an area which size depends on the yield stress of the fluid [17], and can be either symmetric [18] or asymmetric [17], depending on the sample preparation. Similar results had been found for Laponite, a very thixotropic colloidal suspensions [19], where the asymmetry of the flow pattern increases with the age of the fluid through the appearance of a negative wake (i.e. upward fluid motion in the sphere's wake [20]) and in viscoelastic aqueous polyacrylamide solutions [21]. Another peculiar characteristic of a falling sphere in yield stress fluids or in viscoelastic materials is that its velocity can depart from a constant value and show oscillations and irregularities. One of the first observations of an oscillating particle settling has been in hydroxyl propyl guar (HPG) polymer gels [22], a yield-stress power-law family of fluids [23]. In this work, the authors suggested that the irregularities in the descending motion were linked to the elasticity of the gel. Something similar is reported in entangled wormlike micellar fluids [24, 25], in Laponite [26], where the vertical velocity 
can oscillate in bursts and, again, in HPG [27]. In cornstarch suspensions $[28,29]$, the sphere velocity never approaches a steady terminal velocity but instead it oscillates, decreases with time, to reach a series of stop-go cycles as the sphere becomes close to the experimental tank bottom. In wormlike micellar fluids, the cause of non-transient oscillations is to be found in the formation and successively breakage of flow-induced structures; in cornstarch suspensions, in the formation and dissolution of a jammed layer that, under sufficient stress, increases drag and slows the sphere down; while the irregular motion in laponite is due to the existence of flow instabilities and shear banding.

In this paper, we consider viscoplastic fluids that are constituted of an aqueous suspension of soft gel grains. To do so, we place ourselves in between the two end-members described above, using macroscopic super absorbent gels. Varying independently the size of the gels grains and the size of the falling spheres allows us to investigate the influence of the particle size on the effective rheology of the fluid and on the motion of spheres that are falling through it. The paper is organized as follow: in section 2 we describe the experimental set-up and the fluids used during the experiments. Section 3 presents the different regimes of motions that were observed. We close with section 4 and section 5 where results and their implications are discussed and summarized. 


\section{Experimental set-up and fluids}

\subsection{The fluids}

According to the product company (Omiya Green Service Co., Ltd), the fluid used is a superabsorbent polymer (polyacrylamide) made by copolymerization of acrylic acid and acrylamide. It is part of the family of aqueous dispersions of superabsorbent polymers (SAPs), polymeric materials with large capacity of water absorption [30, 31] which are used in many different water absorbing applications in agriculture [32], in health care industry (e.g. in sanitary pads and baby diapers), in sealants and in air-fresheners $[33,34]$. The dry material is a white and granular powder of particles with different irregular shapes. In water, the original small particles can swell up to 200 times and form gel grains whose size can be controlled by controlling the size of the initially dry grains. Beside the general behaviour of such materials that combines viscoelastic [31] and shear-thinning aspects [35], the measurement of appropriate rheological properties at the swollen state is still quite complicated as typical commercial SAPs have large (up to few millimeters) and irregular swollen particles that render classical rheological methods difficult to use [31].

Starting from the provided raw material as the largest grains size endmember, we gradually ground the dry particles to form four thinner powders. All the final gel samples were then obtained by letting $17.5 \pm 0.1 \mathrm{~g}$ of dry product react with $3.5 \mathrm{l}$ of distilled water for one night. The resulting swollen mixture have been gently stirred (100 rpm) for 2-3 days with an electric stirrer in order to remove air bubbles and to homogenize the mixture. Careful weight measurements of a given volume of the final aqueous suspension before and 

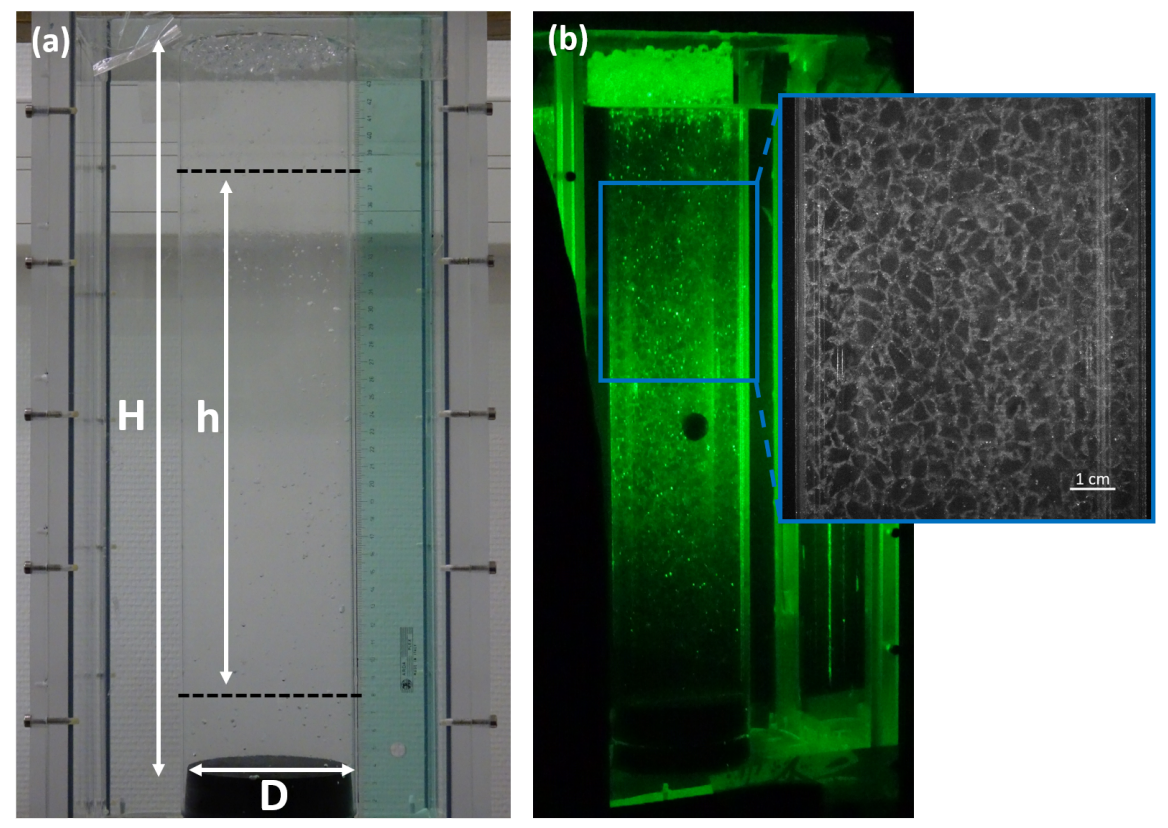

Figure 1: (a) Experimental set up. The cylindrical vessel containing the fluid is $50 \mathrm{~cm}$ high $(H)$ and $10 \mathrm{~cm}$ wide $(D)$. Videos are recorded in the tube section between the two black dotted lines $(h)$. (b) Black and white image of the fluid structure illuminated by a laser sheet. The gel grains are in dark grey and the thin water film around them is in light grey.

after it was completely drained from the free water allowed to estimate a particle volume fraction of $62 \pm 2 \%$.

As it was for the starting dry powders, the five final fluids under scrutiny differ from each other only in the size of the grains that make up their structure. To obtain the grains size distribution of the swollen gel samples, we analyzed several high resolution images (4288 x 2848 pixels) of a laserilluminated vertical cross-section of the experimental tank (Fig.1) by using Multispec, an image analysis software [36]. The sample was stirred between each image, so that each image shows a different organization of the fluid 


\begin{tabular}{lcccc}
\hline name & $\begin{array}{c}\text { Mean diameter } \\
(\mathrm{mm})\end{array}$ & $\begin{array}{c}\text { std } \\
(\mathrm{mm})\end{array}$ & $\begin{array}{c}\text { Median } \\
(\mathrm{mm})\end{array}$ & $\begin{array}{c}\text { Max diameter } \\
(\mathrm{mm})\end{array}$ \\
\hline Gel A & 1.4 & 0.3 & 1.4 & 2.3 \\
Gel B & 2.1 & 0.5 & 2.1 & 3.5 \\
Gel C & 3.3 & 0.9 & 3.0 & 5.8 \\
Gel D & 4.8 & 1.2 & 4.5 & 9.5 \\
Gel E & 5.7 & 1.2 & 5.5 & 9.6 \\
\hline
\end{tabular}

Table 1: Results from the imaging analysis of our five gel samples. Here we report the mean diameter of the grains, the standard deviation (std), the median and the maximum diameter. The grain equivalent diameter $\left(d_{g}\right)$ of a specific gel sample refers to the mean grain size value.

structure. Fig.1b shows an example of how the fluid structure looks like. The arrangement of the grains is easily recognizable and the sampling of each of them during the images analysis can be automatically or manually done directly from black and white images without needing more complex adjustments. Once the outline of the grains is defined from the image, the grain surface comes by counting the number of pixels of which it is composed. We describe each grain by the equivalent diameter of a circle with the same area as the measured surface. The final grains size distributions are reported in Fig.2 where data are normalized to a probability density function and in Table 1 where the characteristics of the distributions are listed. 

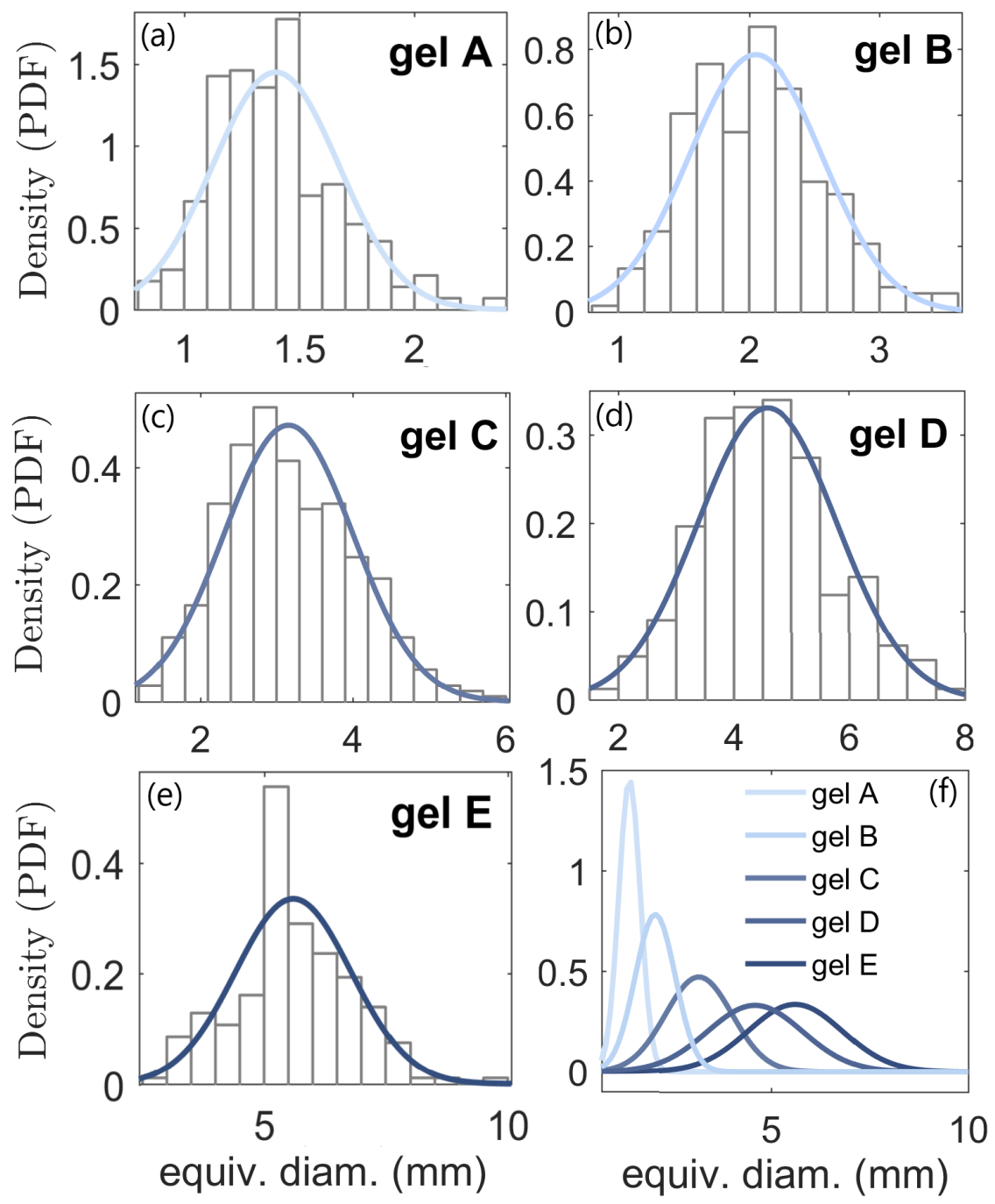

Figure 2: Grains size distributions for the five gels used in the experiments. Histogram representation of grains equivalent diameters with a normal distribution curve on top for gel A (a), gel B (b), gel C (c), gel D (d) and gel E (e). (f) All gels together. 


\subsection{Setup}

All the spheres are released in a $50 \mathrm{~cm}$ deep $(H)$ and $10 \mathrm{~cm}$ wide $(D)$ cylindrical Plexiglas vessel, previously filled with approximately 3.5 liters of fluid (Fig.1a). In order to reduce optical distortions during the recording of images illuminated laterally by a laser sheet, the vessel was placed in a rectangular tank filled with water. A vertical cross-section of the tank is then illuminated by a $532 \mathrm{~nm}$ laser sheet, and images are recorded using a video camera (Canon Legria HF S21 1080p) with frame rate of 25 frames/s. The position of the center of the sphere on each frame was determined by plotting the spatio-temporal evolution of a vertical pixel line centered on the sphere, or by using the blob analysis method in Matlab [37] (fig.4 and 5a). Both methods allow the derivation of the local vertical velocity of the sphere through time. And the blob analysis also provides the measurements of the horizontal position and velocity of the sphere.

We run experiments with spheres of different materials and sizes. Their properties (i.e. density and diameter) are summarized in Table 2. All the steel spheres (density of 7980 and $7970 \mathrm{~kg} / \mathrm{m}^{3}$ ) have been painted with black spray paint to avoid strong reflections, while the other spheres have been left with their original surfaces. The use of spray paint could have increased the surface roughness that in turn can affect the velocity field in the fluid around the object as well as the shape of the yielded region [38]. However, we did not observe any discrepancies between the painted and the bare spheres.

Before each experiment, we stirred gently the fluid to remove any possible preferential path that might have been formed during previously runs. SAP, in fact, shows a quite strong hysteresis where the fluid structure does not 


\begin{tabular}{cc}
\hline$\rho_{s}\left(\mathrm{~kg} / \mathrm{m}^{3}\right)$ & $d_{s}(\mathrm{~mm})$ \\
\hline 14952 & 6.00 \\
7980 & $6.00 ; 12.00 ; 14.00 ; 16.00 ; 18.00$ \\
7970 & $3.00 ; 4.00 ; 5.00 ; 7.00 ; 8.00 ; 10.00 ; 20.00 ; 22.00 ; 25.00$ \\
7799 & 6.00 \\
7782 & 14.00 \\
7621 & 8.00 \\
7519 & 3.20 \\
3227 & 6.00 \\
2200 & $15.66 ; 15.81$ \\
\hline
\end{tabular}

Table 2: Spheres density $\rho_{s}$ and diameter $d_{s}$.

recover completely its original state after it has been deformed. This aspect is observed when a second sphere is dropped from the same position as the previous one without stirring the fluid in between the two runs. For identical spheres, we have always measured higher velocities for those released later and falling through an already deformed path. Stirring the fluid between runs prevents this issue and allowed us to get reproducible velocities for identical spheres.

\section{Experimental results}

As first step of our investigation, it is useful to test the reproducibility and the accuracy of the experimental techniques and possible limits arising from the dimension of our setup. To do so, we measured the terminal ve- 
locities $\left(v_{y}\right)$ of spheres settling in a Newtonian fluid at $T_{c}=15.2 \pm 0.3{ }^{\circ} \mathrm{C}$. The fluid is glucose syrup (Glucor $60 / 80$ ) with $\rho=1394.66 \mathrm{~kg} / \mathrm{m}^{3}$ and $\eta=12.3 \pm 0.7$ Pa.s, density and viscosity at $T_{c}$, respectively. Spherical objects falling in a Newtonian fluid at low Reynolds number reach a terminal velocity given by the Stokes velocity

$v_{\text {Stokes }}=\frac{2}{9} \frac{R^{2}\left(\rho_{s}-\rho_{\text {fluid }}\right)}{\eta} g$

where $R$ is the radius of the spherical object and $g$ the acceleration due to gravity. In Fig.3 we plot the velocities measured for spheres of same density and diameters in between 3 and $18 \mathrm{~mm}$. Larger spheres are subjected to wall effects and in this case the sphere terminal velocity can be corrected by a coefficient that writes as

$$
K_{1}^{-1}=1+\alpha_{1}\left(\frac{d_{s}}{D}\right)+\alpha_{2}\left(\frac{d_{s}}{D}\right)^{3}+\alpha_{3}\left(\frac{d_{s}}{D}\right)^{5}
$$

$$
\text { where } \alpha_{1}=-2.104443, \alpha_{2}=2.08877, \alpha_{3}=-0.94813 \text { and } D \text { is the tube }
$$
diameter [39]. Experiments carried out in syrup show that the measured settling velocities are in good agreement with relative corrected Stokes velocities, that is $v_{y} /\left(v_{\text {Stokes }} K_{1}^{-1}\right)=1$, ensuring that our measurement system is accurate (Fig.3).

We now move to the case where the fluid through which spheres fall is one SAP sample described in the previous section. In Fig.4 we report a selection of falling profiles in gel $\mathrm{C}$ (grains mean diameter $d_{g}=3.3 \mathrm{~mm}$ ) for four falling spheres with same density but different diameters. The same type of plot is also showed in Fig.5a where this time we track spheres having same size 


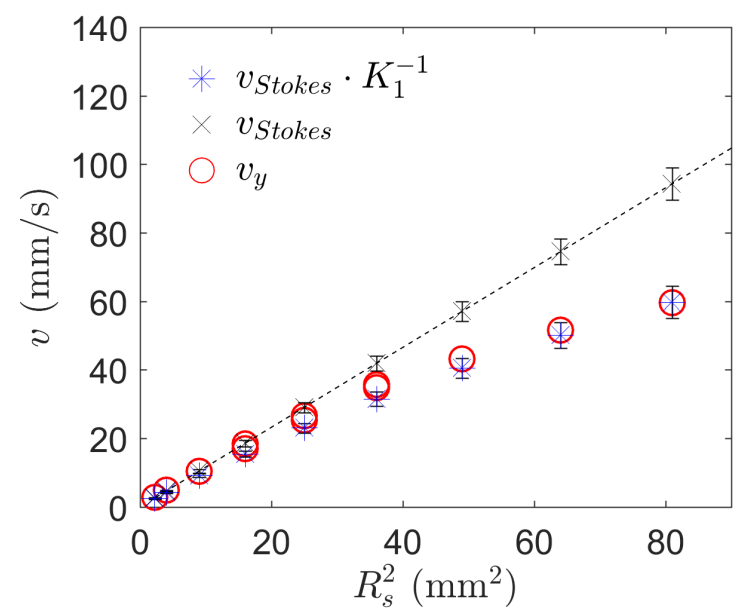

Figure 3: Measured terminal velocities, $v_{y}$, in syrup (circles) and Stokes velocities, $v_{\text {Stokes }}$, (crosses and dashed line) as function of spheres squared radius, $R_{s}^{2}$. Uncertainties for Stokes velocities come from the uncertainties in determining the syrup viscosity whereas the error for $v_{y}$ is $0.25 \mathrm{~mm} / \mathrm{s}$, smaller than the symbols. Star symbols are the ideal velocities considering the wall correction, eq. (2). 


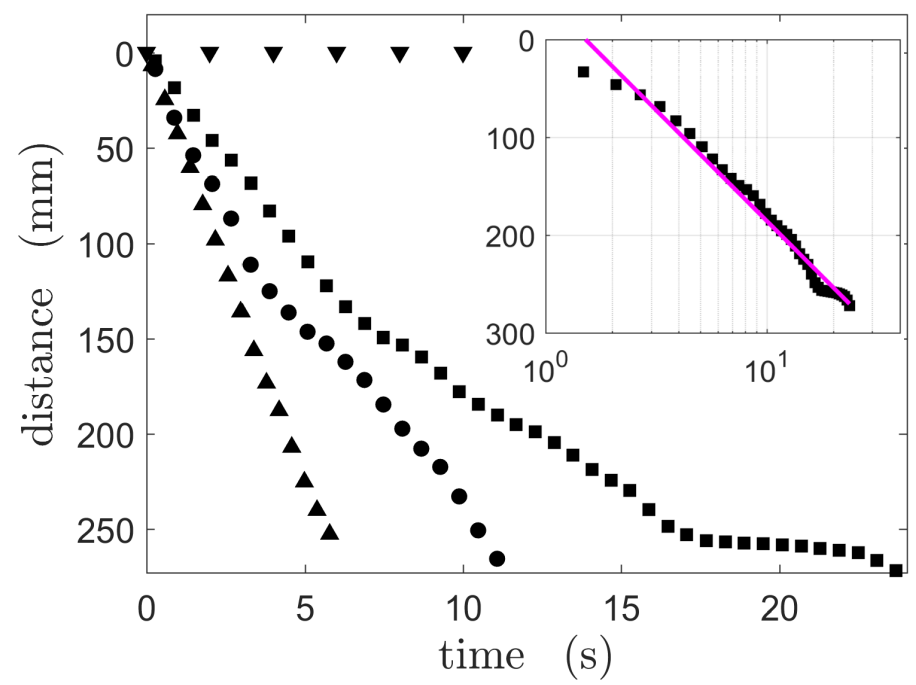

Figure 4: Depth of spheres settling through gel $\mathrm{C}$ as function of time. Spheres have constant density $\left(\Delta \rho=6980 \pm 10 \mathrm{~kg} / \mathrm{m}^{3}\right)$. Symbols refer to the diameter of the sphere: $d_{s}=10 \mathrm{~mm}(\boldsymbol{\Lambda}), d_{s}=8 \mathrm{~mm}(\bullet), d_{s}=7 \mathrm{~mm}(\boldsymbol{\square}), d_{s}=4 \mathrm{~mm}(\mathbf{\nabla})$. Varying sphere's diameter, one can recognize the following motion regimes: linear regime (upward-pointing triangles), irregular motion superimposed to a linear trend (circles), logarithmic regime (squares) and no-motion (downward-pointing triangles). Regimes (1), (2), (4) and (5) in the text, respectively. The insert shows the distance as function of time for regime (4) with a semi-log scale. The color line is the fit $y(x)=a \log (b x)$, where $a=98.34$ and $b=0.66$. The correlation coefficient is $\mathrm{R}=0.980$.

but different densities settling in gel $\mathrm{D}\left(d_{g}=4.8 \mathrm{~mm}\right)$. In both figures, the starting position along $\mathrm{y}$-axis, $h=0$, does not refer to the top fluid surface but to the upper limit of the camera view at which movies were recorded (Fig.1a). This is a consequence of the fact that all movies have been taken for a magnified portion of tube $h=300 \mathrm{~mm}$, starting from $70 \mathrm{~mm}$ under the surface. Camera position and therefore size of the view are kept constant for each run done in the same fluid. 
(a)

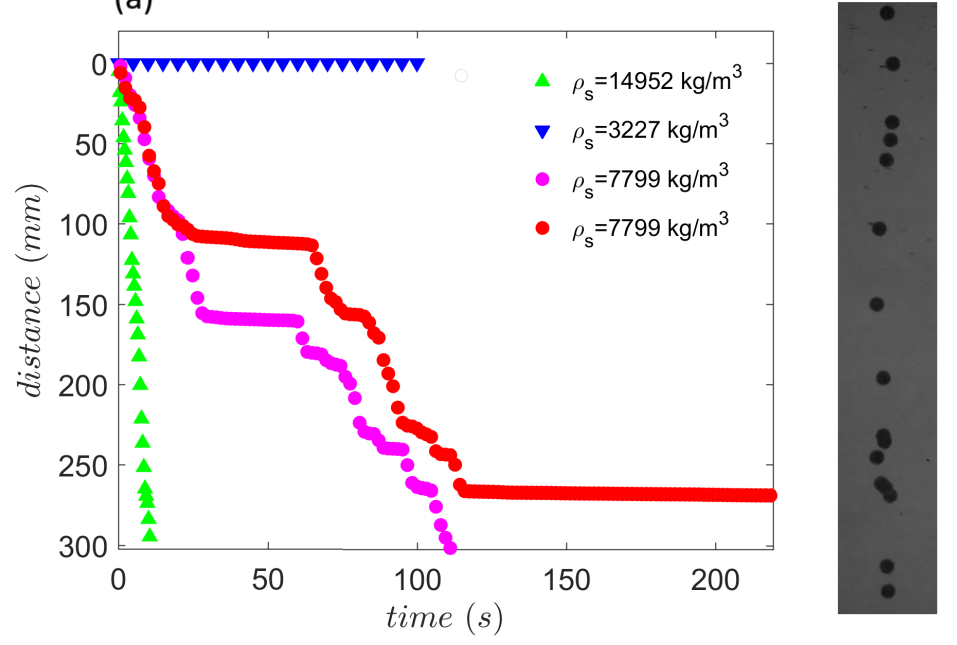

Figure 5: (a) Depth of spheres settling through gel D as function of time. Here spheres have constant diameter $\left(d_{s}=6 \mathrm{~mm}\right)$ but different densities. As in Fig.4, we report the case of linear regime (upward-pointing triangles), no-motion (downward-pointing triangles) and, in addition, the intermittent regime (circles). (b) Sequence of snapshots every 100 frames showing the irregular motion in gel $\mathrm{D}$ for a sphere with $d_{s}=7 \mathrm{~mm}$ and $\rho_{s}=7970 \mathrm{~kg} / \mathrm{m}^{3}$. 
For each gel, depending on the diameter and density of the sphere, we distinguish the following five regimes (Fig.4 and Fig.5). (1) A linear regime: rapid and linear fall in which spheres reach a constant terminal velocity; (2) an irregular regime superimposed to a linear one where spheres never stop during their way down $\left(v_{y}>0\right)$ but their local velocity fluctuates around a constant value; (3) an intermittent regime: another irregular regime but here periods of no-motion, where the sphere's vertical velocity goes to zero, and periods of irregular falls follow one another; (4) a logarithmic regime where the sphere's speed progressively decreases (inset in Fig.4); and (5) a no-motion regime in which spheres do not move at all from their initial positions.

Regimes (1) and (5) are characteristic of a yield stress fluid [11, 26, 40] and one can interpret them based on the state of the medium that surrounds the object. In the linear regime (1) the fluid around the sphere has a liquidlike behaviour that allows spheres to go down, whereas in regime (5) local stresses are below the yield stress $\left(\sigma_{Y}\right)$ and the material around the sphere remains in the solid state, leading to the no motion of the object. Here the stress field is generated by the weight of the sphere minus the buoyancy and the no-motion regime is always observed for smallest diameters $d_{s}$ (Fig.4) or density contrast $\Delta \rho$ (Fig.5a, as in [11]). We have had no-motion whether the sphere was placed on the surface of the fluid column, or it was gently pushed within it and released deeper in the fluid. In both cases, we left the spheres in that position for days (up to a week for one run) and they never moved away from their starting positions.

Although the distinction between the two end-member cases (1) and (5) 
is easy to establish, to discern quantitatively between the first steady-state motion regime (1) and the more irregular ones is more delicate. We summarize how we define the different motion regimes in Table 3. From the falling profiles one can notice that, although sometimes it is still possible to identify an almost constant falling speed superimposed on fluctuations (circle symbols both in Fig.4 and Fig.5a), in some other cases, spheres seem to slow down logarithmically during their way to the bottom (square symbols in Fig.4), making impossible the determination of a constant terminal velocity. We report this aspect in Fig.6a by plotting the mean squared variations (MSE) obtained by fitting the falling profiles with a linear or a semilog fit as function of spheres buoyancy. The logarithmic regime (4) is defined for $\mathrm{MSE}_{\text {linear }}>\mathrm{MSE}_{\text {log }}$, that is when falls are better represented with a logarithmic fit. Hence, this regime describes a continuous decrease in sphere's speed which never reaches the steady-state. Although in the case of Carbopol [11] velocity decreases following a power law, in most of our cases it best fits a logarithmic curve. Although from Fig.6a is evident that the logarithmic behaviour appears more often at lower buoyancies, the distinction between linear and non-linear regime is not straightforward. In a few cases, indeed, at same buoyancy the two behaviours coexist.

Once the separation between stoppage cases (logarithmic regime (4) and no-motion (5)) and the other cases is established, one can identify and recognize diversities between the steady-state motion (regime (1)) and the more irregular and chaotic regimes (2) and (3). Depending on the buoyancy of the object and hence on its velocity, the irregularities show up as fluctuations in the sphere local vertical velocity and in a progressive increase of its 

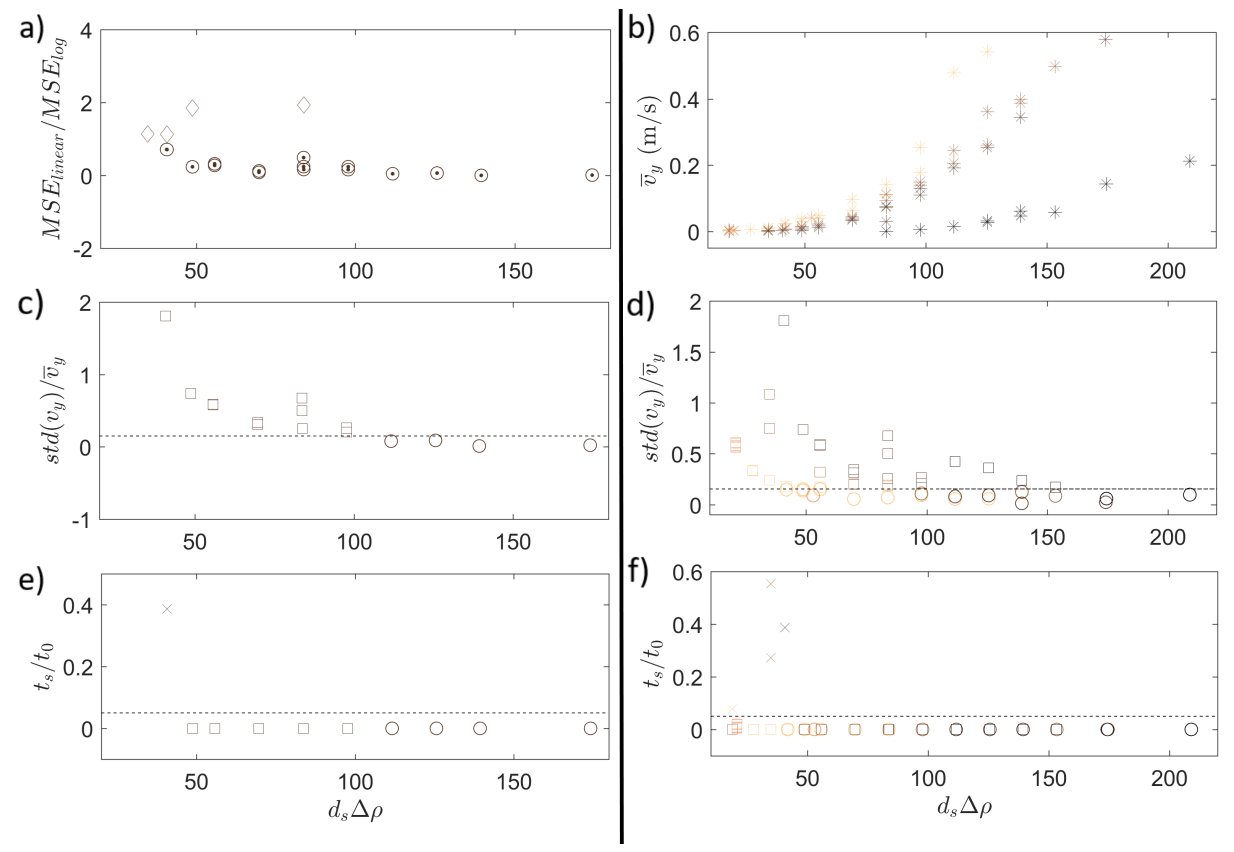

Figure 6: Determination of the different regimes of motion. In (a), (c) and (e) data refer to experiments carried out in gel E. In (b), (d) and (f) data are for all fluids and colours indicate the grain size as in Fig.2. (a) best fit $\left(M S E_{\text {linear }} / M S E_{\text {log }}\right)$ of the falling profiles. $\odot$ indicates a better linear fit whereas $\diamond$ a better semilog fit (logarithmic regime (4), in the text). (b) mean falling velocities $\left(\bar{v}_{y}\right)$ for those falling profiles that have been linearly fitted. The error for $v_{y}$ is $0.25 \mathrm{~mm} / \mathrm{s}$, smaller than the symbols. We do not report the standard deviation for $v_{y}$ here (whereas it is in (c) and (d)) as being also linked to the physical fluctuation of the sphere, for small values of velocity it would make the plot unreadable. In (c) and (d) we separate those experiments that show a more irregular and chaotic motion $(\square)$ from those that do not show any irregularities during the fall $(\bigcirc)$ by plotting $\operatorname{std}\left(v_{y}\right) / \bar{v}_{y}$. The dashed line is for $\operatorname{std}\left(v_{y}\right) / \bar{v}_{y}=0.15$, from the Newtonian case. In $(e)$ and $(f)$ we separate the intermittent regime $(3)(\times)$ from the other irregular regime (2) ( $\square$ ) based on the amount of time $t_{s}$ the sphere remains at $v_{y} \leqslant \operatorname{err}\left(v_{y}\right) . t_{0}$ is the total time the sphere takes to descend $h$. The dashed line indicates $t_{s} / t_{0}=5$. 
horizontal motion (Fig.5b). Regimes (2) and (3) show some similarities: in both of them, spheres have a long term constant falling velocity on which is superimposed a more complex and irregular pattern of fluctuations. The latter are characterized by accelerations and subsequent decelerations in a short period of time and, in some cases, they can result in a succession of stops of the sphere. In the most drastic cases, we observe the complete arrest of the sphere after it has passed through most of the fluid (Fig.5a).

To determine the boundaries between the irregular regimes (2) and (3) and regime (1), one can look, as a first approximation, at the ratio between the standard deviation (std) of the local falling velocity and its mean value. Variations of the local velocity for spheres in irregular regimes are much larger than the experimental uncertainty (Fig.6c-d). The latter is due to the precision of the technique we used to detect the sphere position in time (hereafter $\left.\operatorname{err}\left(v_{y}\right)\right)$. It is estimated to be $0.25 \mathrm{~mm} / \mathrm{s}$ from the syrup-case measurements and from local velocity measurements in the no motion regime. In the Newtonian case, $\operatorname{std}\left(v_{y}\right) / \bar{v}_{y}$ always remains smaller than 0.15 . We define the linear regime (1) using this value, that is for $\operatorname{std}\left(v_{y}\right) / \bar{v}_{y}<0.15$ (dashed line in Fig.6c-d). This limit represents therefore a boundary between the steady-state motion and what shows a more spread distribution of velocities.

Once the linear regime is bounded in this way, we distinguish between the irregular regime (2) and the intermittent regime (3) as follows. In the intermittent case the sphere remains during a certain cumulative amount of time, $t_{s}$, at $v_{y} \leqslant \operatorname{err}\left(v_{y}\right)$. We then compare $t_{s}$ to the total time, $t_{0}$, the sphere takes to descend $30 \mathrm{~cm}$. The intermittent regime is defined as $t_{s} / t_{0} \geq 5 \%$. The remaining irregular regime (2) is therefore defined as $t_{s} / t_{0}<5 \%$ and 


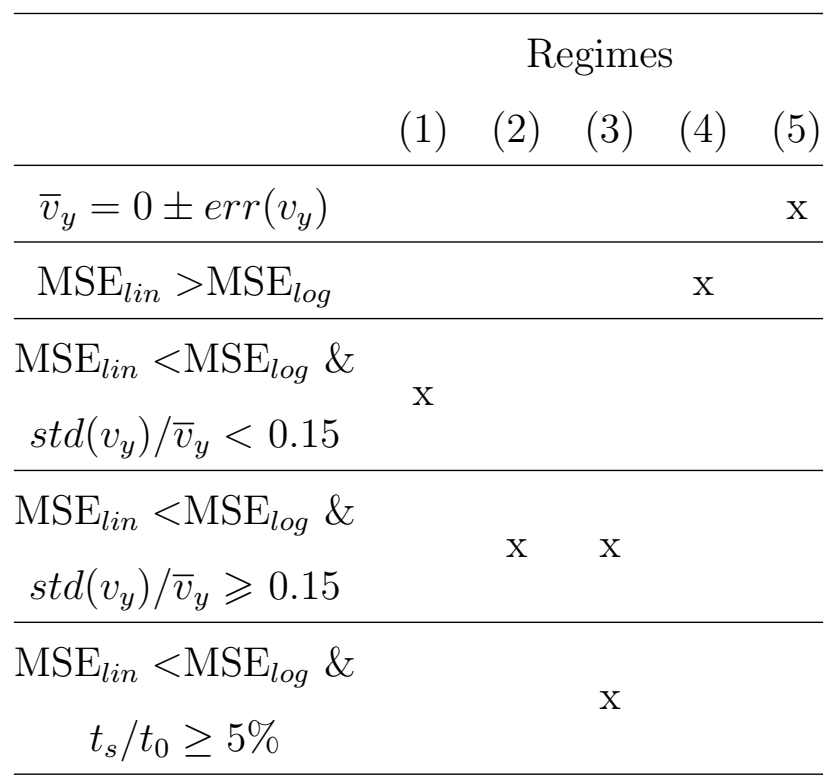

Table 3: Summary of the observed motion regimes and the way they are classified

$\operatorname{std}\left(v_{y}\right) / \bar{v}_{y}>0.15$ (Fig.6e-f).

Fluctuations, accelerations and subsequent decelerations and the resulting general complex set of behaviours of the moving sphere, characterize the experiments both in the direction of fall and in the one perpendicular to it. In fact, the irregular motion we have observed is not only present and visible in the vertical direction but it also affects the horizontal motion of spheres in a such a way that, along this direction, they collect a certain amount of traveled distance $\left(L_{x}\right)$ with respect to their initial position, depending on their diameter (Fig.7a), on the gel grains size (Fig.7b), and on the vertical falling distance $h$ (Fig. 8a). Fig. 8b shows that for each run, $L_{x}$ is roughly proportional to $h$. Fig.7 shows that small spheres change frequently their horizontal position, oscillating continuously around the vertical axis, and traveling for larger distances $L_{x}$ than the large spheres. If we normalize $L_{x}$ by $h$ and $d_{s}$ 

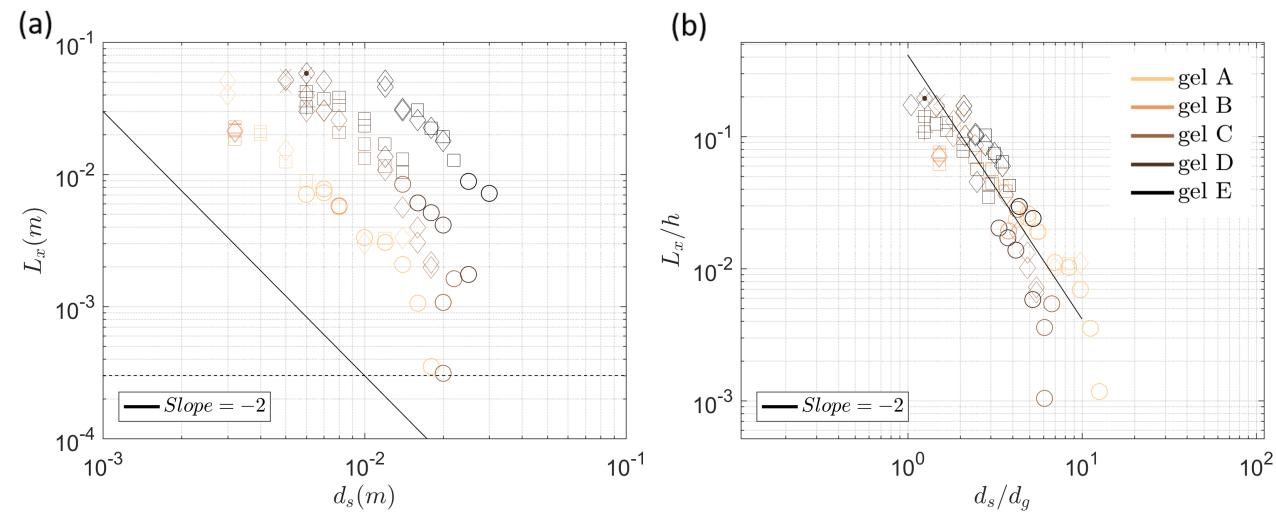

Figure 7: Total distance traveled in the horizontal direction $\left(L_{x}\right)$. (a) $L_{x}$ as function of the sphere diameter $d_{s}$. Dashed line indicates the uncertainty in position. (b) Same plot with y-axis normalized by the vertical falling distance $h$ and x-axis by the gel grain size $d_{g}$. Colours indicate the different fluids as indicated in the legend. Symbols refer to the different motion regimes: $\bigcirc$ for the linear regime (1); $\square$ for an irregular regime superimposed to a linear one $(2) ; \times$ for the intermittent regime $(3)$ and $\diamond$ for the logarithmic regime (4). Empty symbols are for steel spheres while the symbol with a point inside refers to $\rho_{s}=7799 \mathrm{~kg} / \mathrm{m}^{3}$ and those filled with a plus sign are for $\rho_{s}=14952 \mathrm{~kg} / \mathrm{m}^{3}$.

by the diameter of the gel grains $\left(d_{g}\right)$, we achieve a good collapse for all our data along a line with slope -2 . That is

$$
L_{x} / h=C_{\exp }\left(d_{s} / d_{g}\right)^{-2} .
$$

where $C_{\text {exp }}=0.49 \pm 0.26$. In this way the total travelled distance in horizontal direction turns to be a good candidate to underline the strong interaction of the sphere and the gel grains that make up the fluid structure when those two have similar sizes. 

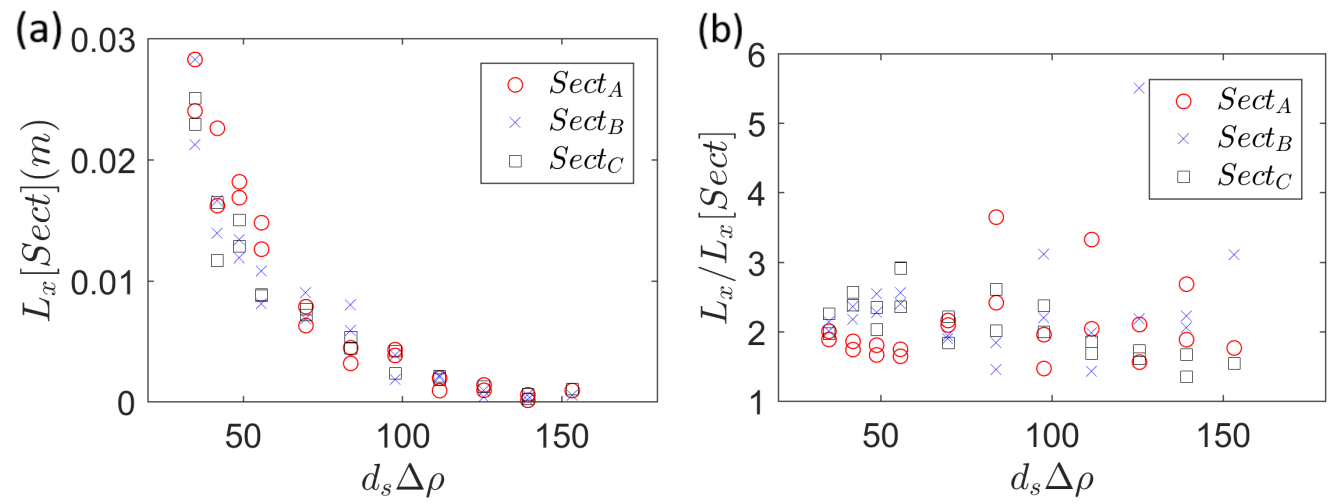

Figure 8: Distance traveled in the horizontal direction in gel C. (a) Distance traveled in different 15-cm-vertical-sections of $h, L_{x}[$ Sect $]$, as function of spheres buoyancy. Sections are Sect $_{A}=[h=0 \mathrm{~cm}, h=15 \mathrm{~cm}] ;$ Sect $_{B}=[h=15 \mathrm{~cm}, h=30 \mathrm{~cm}] ;$ Sect $_{C}=[h=7.5 \mathrm{~cm}, h=22.5$ $\mathrm{cm}$. (b) Ratio between the total distance traveled in $30 \mathrm{~cm}, L_{x}$, and the distance travelled in $15 \mathrm{~cm}, L_{x}[$ Sect $]$.

\section{Discussion}

\subsection{Determination of the SAP effective rheology}

The fall of a spherical object within certain boundary conditions is described by the combination of a proper set of equations of motion and the constitutive relation of the fluid through which the sphere is falling. For yield stress fluids a commonly used rheological model that relates stress and strain in a non-linear way is the Herschel-Bulkley model [2, 12, 41]:

$$
\begin{cases}\sigma=\sigma_{Y}+K_{v} \dot{\gamma}^{n} & \text { if } \quad \sigma>\sigma_{Y} \\ \dot{\gamma}=0 & \text { if } \quad \sigma \leq \sigma_{Y}\end{cases}
$$

where $\sigma$ is the stress, $\sigma_{Y}$ the yield stress, $\dot{\gamma}$ the shear rate, $K_{v}$ the consistency and $n$ the shear-thinning index. Considering a fluid which flows following 
such model, for small Reynolds numbers $(\mathrm{Re} \ll 1)$, the problem of a settling particle needs two parameters to be characterized [11, 42]. They are the Bingham number

$$
B i=\frac{\sigma_{Y}}{K_{v}\left(v_{y} / d_{s}\right)^{n}},
$$

that compares yield stress with viscous stresses, and the yield number

$$
Y=\frac{3 \sigma_{Y}}{g d_{s} \Delta \rho}
$$

that compares the yield stress with the buoyancy stress.

As described in section 1, most of the experimental work done to study this problem is carried out by using Carbopol. The main difference between experiments done with Carbopol and the present work regards the typical size of the fluid structure compared to the dimension of the object that is moving through it. Carbopol corresponds to the case where the fluid structure (grains) is much smaller than the spheres. In this case, only regimes (1), (4) and (5) were reported (e.g. [11]) while neither the irregular regime (our regime (2)) nor the intermittent regime (3) were reported; and the fall of the sphere is always reported to be free of any sort of chaotic motion $[11,17,38]$.

In regime (1), that is when a constant terminal velocity can be determined, Tabuteau et al. [11] showed that their data are well-fitted by the following equation

$$
\frac{1}{Y}=7+\frac{8.52}{B i}
$$


with $n=0.5$, and that there is a critical value of $Y, Y_{c}=0.145$, above which there is no motion.

Taking into account the differences between SAP and Carbopol, one can use eq.(7), defined for $d_{s} \gg d_{g}$, and check whether it applies for a fluid with a much larger structure. Thus, assuming a framework in which eq.(7) is valid also in our case, we can rewrite it as

$$
\frac{g d_{s} \Delta \rho}{3}=7 \sigma_{Y(e f f)}+8.52 K_{v(e f f)}\left(\frac{v_{y}}{d_{s}}\right)^{n},
$$

where $\sigma_{Y(e f f)}$ and $K_{v(e f f)}$ represent the effective yield stress and consistency of the SAP through which the sphere is falling. In eq.(8), $v_{y}$ is the average falling velocity defined for those experiments which do not show a decrease in speed, that is those in regime (1), (2) and (3). By plotting the buoyancy stress $\left(g d_{s} \Delta \rho\right)$ as function of the effective strain rate, i.e. $\left(v_{y} / d_{s}\right)^{n}$, Fig. 9 shows for SAP the same linear relationship showed for Caropol [11]. Therefore the slope of the linear fit is related to an effective consistency through

$$
K_{v(e f f)}=\left[\frac{g d_{s} \Delta \rho}{25.56}-0.86 \sigma_{Y(e f f)}\right]\left(\frac{v_{y}}{d_{s}}\right)^{-n} .
$$

and, similarly, an effective yield stress is related to the intercept at zero effective strain rate through

$$
\sigma_{Y(e f f)}=\frac{g d_{s} \Delta \rho}{21}
$$
gello

In this way, by using the falling sphere system as reometer we can define an effective rheology, which has to have same form of eq.(7), for each of our gel sample. Fits in Fig.9 depend on the shear thinning exponent $n$ used to 
calculate the effective shear rate. Minimum mean squared values for linear fit are found for $n$ in between 0.5 and 0.7 (Fig.11). Unless otherwise indicated, in what follows we will use $n=0.5$ since it is with this value that eq. (7) is defined. Interesting, experiments in both regime (1), (2) and (3) can be linear fitted by the same line. Moreover, effective yield stress and consistency increase with the gel grains size (Fig.10).

For experiments involving large spheres, it is opportune to check whether wall corrections are needed or not. For sufficiently small sphere-to-tube diameter ratio, $d_{s} / D$, we do expect no reductions of sphere's terminal velocity. This means that the measured velocity, $v_{y}$, is equal to the ideal terminal velocity of a sphere falling in an unbounded medium, $v_{\infty}$. On the other hand, if $d_{s} / D$ exceeds a critical value, $\left(d_{s} / D\right)_{c r i t}$, velocity decreases leading to $v_{y} / v_{\infty}<1$. The critical diameter ratio for a yield stress fluid writes as [43]

$$
\left(d_{s} / D\right)_{c r i t}=0.055+1.114 Y \quad \text { if } \quad Y \leqslant Y_{c} .
$$

In our case, $d_{s} / D$ is smaller than $\left(d_{s} / D\right)_{\text {crit }}$ as long as $d_{s} / D<0.1$. The influence of walls causes a reduction in speed such that [43]

$$
\frac{v_{y}}{v_{\infty}}=1-1.7\left[\left(d_{s} / D\right)-\left(d_{s} / D\right)_{c r i t}\right] \quad \text { if } \quad d_{s} / D>\left(d_{s} / D\right)_{c r i t} .
$$

Fit For $0.1 \leqslant d_{s} / D \leqslant 0.17$ experiments are above critical conditions and, according to eq. (12), wall effects lead to $0.8<v_{y} / v_{\infty}<1$, that is a maximum decrease of $v_{y} / v_{\infty}$ of less than $20 \%$.

Considering only the experiments with $v_{y} / v_{\infty}>0.8$ and ignoring the others, we recalculate the effective rheology in the same way described above 


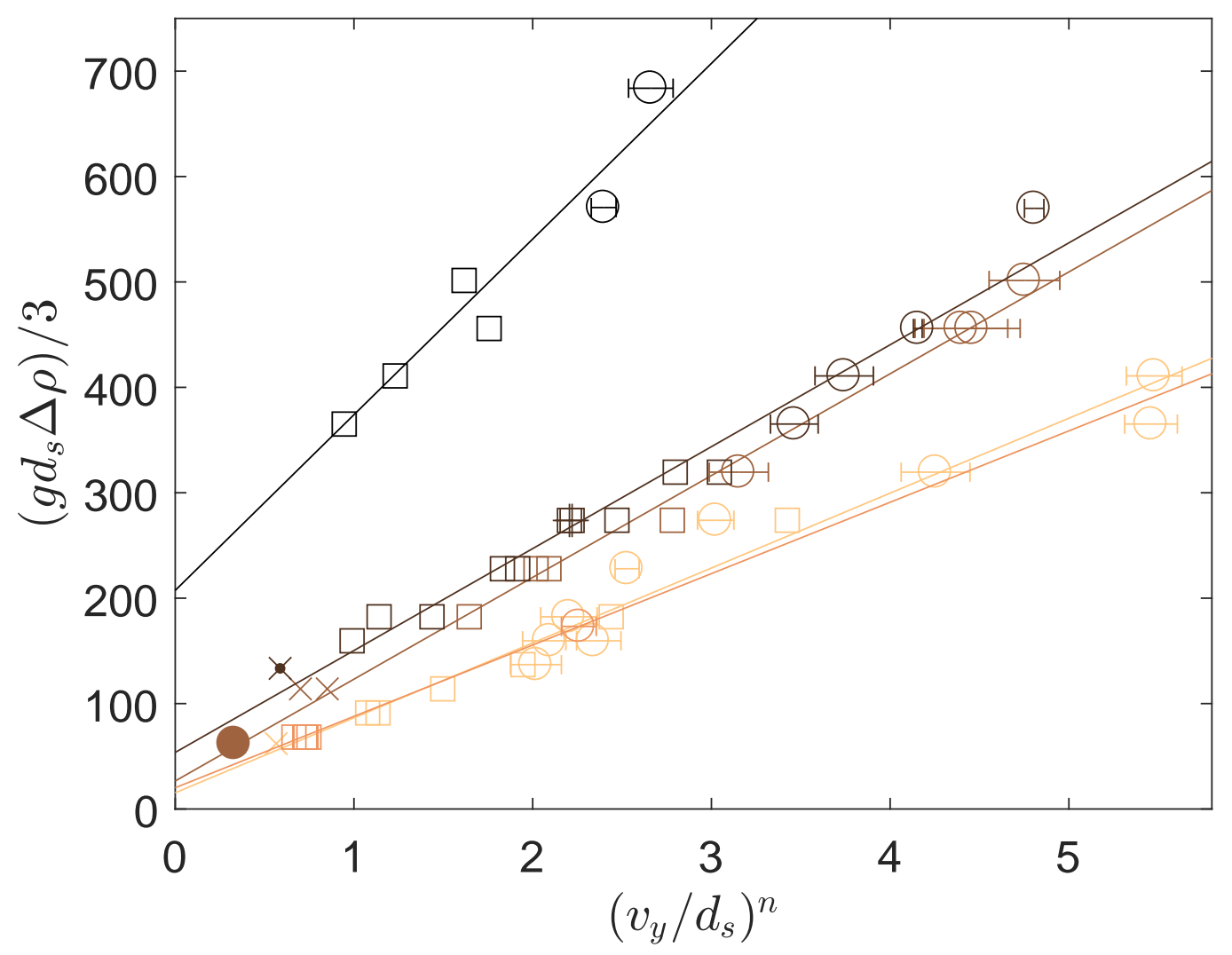

Figure 9: Sphere buoyancy as function of effective shear rate, $\left(v_{y} / d_{s}\right)^{n}$. Following Tabuteau et al. [11] approach, $v_{y}$ is the average falling velocity for those experiments where a steady decrease of the speed has not been observed (i.e. we do not consider regime (4)) and $n=0.5$ the power-law index. Colours represent the fluids (same as Fig.7) while symbols the regimes (circles for regime (1), squares for regime (2) and crosses for regime (3)). Empty symbols for steel sphere; symbol with a point inside for $\rho_{s}=7799$ $\mathrm{kg} / \mathrm{m}^{3}$; with a plus sign for $\rho_{s}=14952 \mathrm{~kg} / \mathrm{m}^{3}$ and filled symbols for $\rho_{s}=2200 \mathrm{~kg} / \mathrm{m}^{3}$. Solid lines are the linear data fits. 


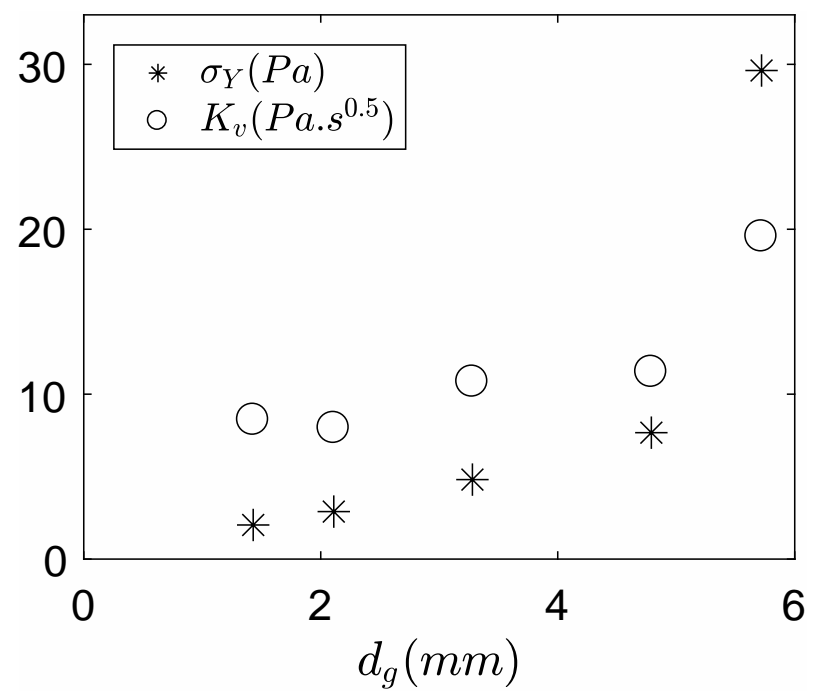

Figure 10: Effective yield stress $\left(\sigma_{Y}\right)$ and effective consistency $\left(K_{v}\right)$ as function of gel grains size.

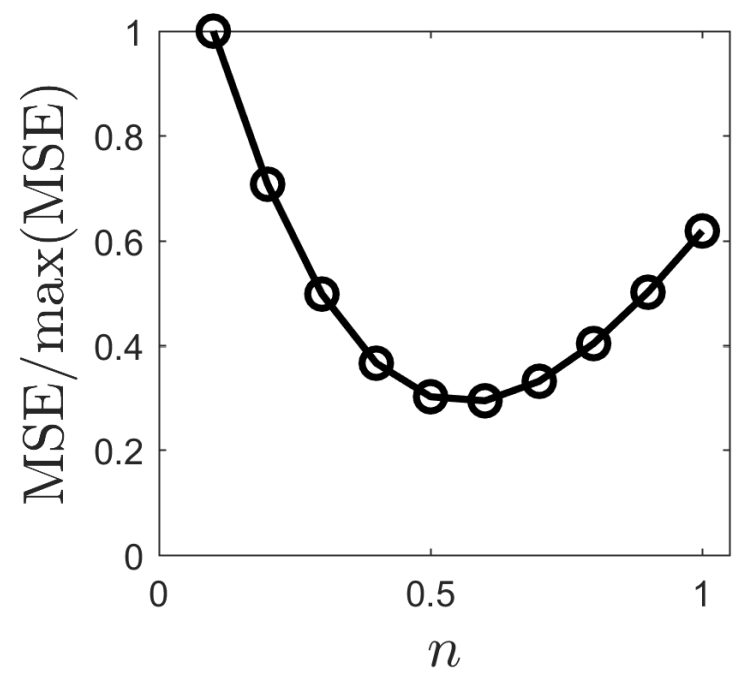

Figure 11: Power law index $(n)$ that best linearly fits data in Fig.9 for all gel samples. $y$ axis represents the mean squared errors (MSE) of the linear fit normalized by the maximum MSE found. The best range of $n$ is in between 0.50 and 0.70 . 

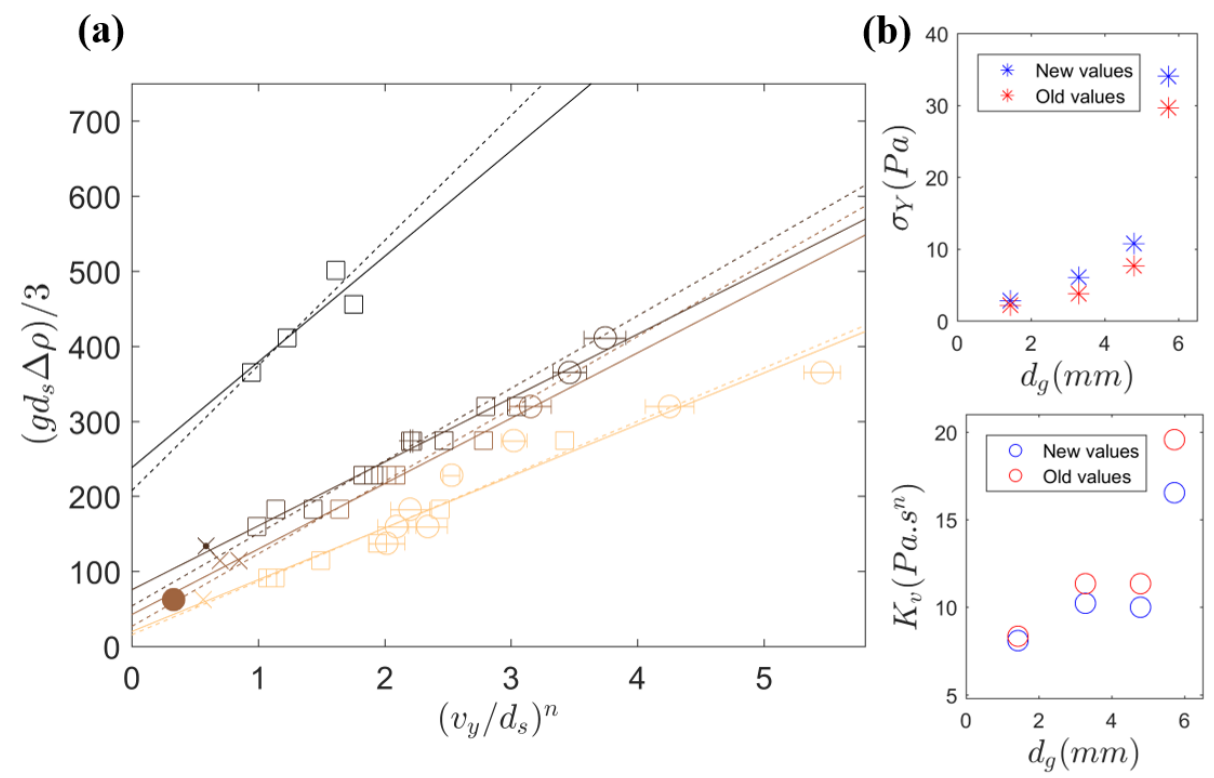

Figure 12: Influence of the velocity reduction due to wall effects on the effective rheology. $n=0.5$. (a) The new fit that considers only experiments with $v_{y} / v_{\infty}>0.8$ (solid lines) is compared with the previous fit from Fig.9 (dashed lines). Colours and symbols here are those described in Fig.9. (b) Effective yield stress and consistency as function of gel grains size from the new fit (blue symbols). Red symbols are those in Fig.10.

(Fig.12a). The new values of $\sigma_{Y(e f f)}$ and $K_{v(e f f)}$ do not differ much from the previous ones (Fig.12b); which means that our set up is big enough.

Once we got the yield stress and the consistency of each sample, we plot $Y^{-1}$ as function of $B i^{-1}$ in Fig.13. For values of $B i^{-1}>0$ we achieve a good collapse along eq.(7) for experimental data that belong to regimes (1), (2) and (3). In this way, even considering the irregular regime (2) and the intermittent (3) where the interaction between moving objects and fluid structure results in highly irregular falls of objects themselves, an effective 
rheology following the Hershel-Bulkley model with a classical shear-thinning index of 0.5 well represents the majority of our data.

\subsection{Entrapment conditions}

Fig.13 also shows that for $B i^{-1}=0$ spheres get entrapped also for $Y^{-1}>$ $Y_{c}^{-1}$. From the experimental work of Tabuteau et al. [11], the critical value of the yield number $\left(Y_{c}\right)$ above which spheres do not move in a simple yield stress fluid is 0.145 . This value is very close to 0.143 , predicted in previous works of Beris et al. [10] and Blackery and Mitsoulis [14]. On the other hand, a more recent study [13] points out how the critical value may increase as function of the Deborah number if elastic effects are taken into account. In our case, however, we observe sphere arrest also for $Y<Y_{c}$. For example, we observed arrest even for $Y=0.042 \approx Y_{c} / 3$ (4 mm steel sphere).

As we highlighted at the beginning of this section, equation (7) was derived for Carbopol when the size of the moving object is much larger than the size of the particles than make up the fluid structure. Inside this assumption, the critical condition is well predicted in our case too. In gel $\mathrm{E}$, for example, we had no-motion for a large glass sphere $\left(d_{s} / d_{g}>2.5\right.$ and $\left.\Delta \rho=1200 \mathrm{~kg} / \mathrm{m}^{3}\right)$ for $Y=0.478$ (Fig.14), greater than $Y_{c}=0.145$. It is only when the diameter of the sphere becomes comparable to the diameter of the gel particles that $Y_{c}$ moves to lower values and the stoppage regime (5) cannot be anticipated any more from eq.(7). For the same gel E, a smaller steel sphere $\left(d_{s} / d_{g}<2\right.$ and $\left.\Delta \rho=6970 \mathrm{~kg} / \mathrm{m}^{3}\right)$ is in stoppage for $Y=0.13<Y_{c}$. We attribute this to the interaction between spheres and the gel structure: when $\mathrm{d}_{s} \rightarrow \mathrm{d}_{g}$, the spheres "see" obstacles on their way and the effective rheology breaks down. 


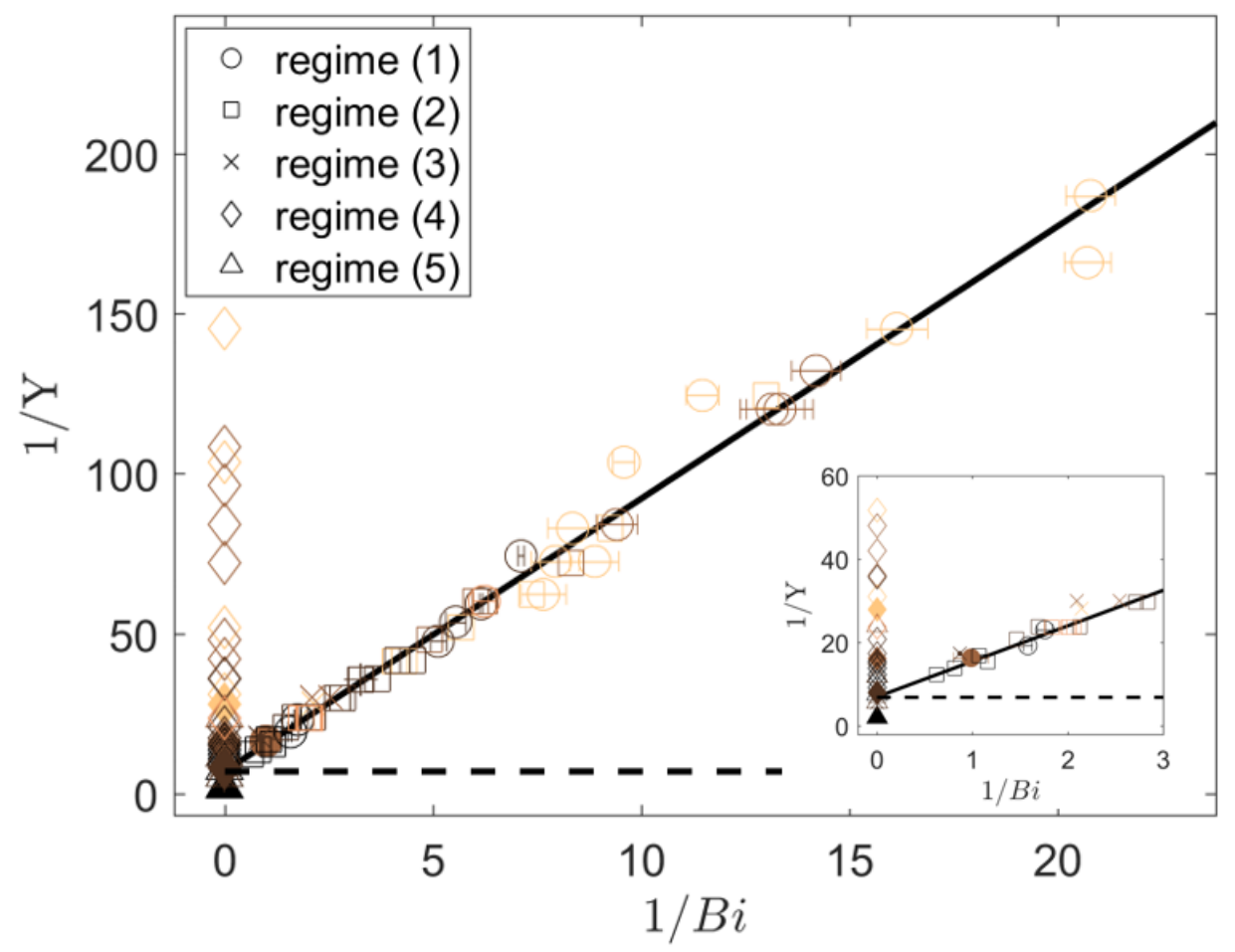

Figure 13: $1 / Y$ as function of $1 / B i$. Colours are for the different fluids. The dashed line indicates $Y_{c}^{-1}=0.145^{-1}$ [11]. Symbols are filled in a different way to distinguish between spheres of different materials: full-filled symbols are for light spheres $\left(\rho_{s}=2200 \mathrm{~kg} / \mathrm{m}^{3}\right)$, open-symbols for $\rho_{s}=7970 \mathrm{~kg} / \mathrm{m}^{3}$, point-filled for $\rho_{s}=7799 \mathrm{~kg} / \mathrm{m}^{3}$, asterisk-filled for $\rho_{s}=3227 \mathrm{~kg} / \mathrm{m}^{3}$ and plus-filled for $\rho_{s}=14952 \mathrm{~kg} / \mathrm{m}^{3}$. We show the good collapse of our data along eq.(7) (solid black line). The inset shows the data zoomed around the critical conditions in order to better display the entrapment conditions. 


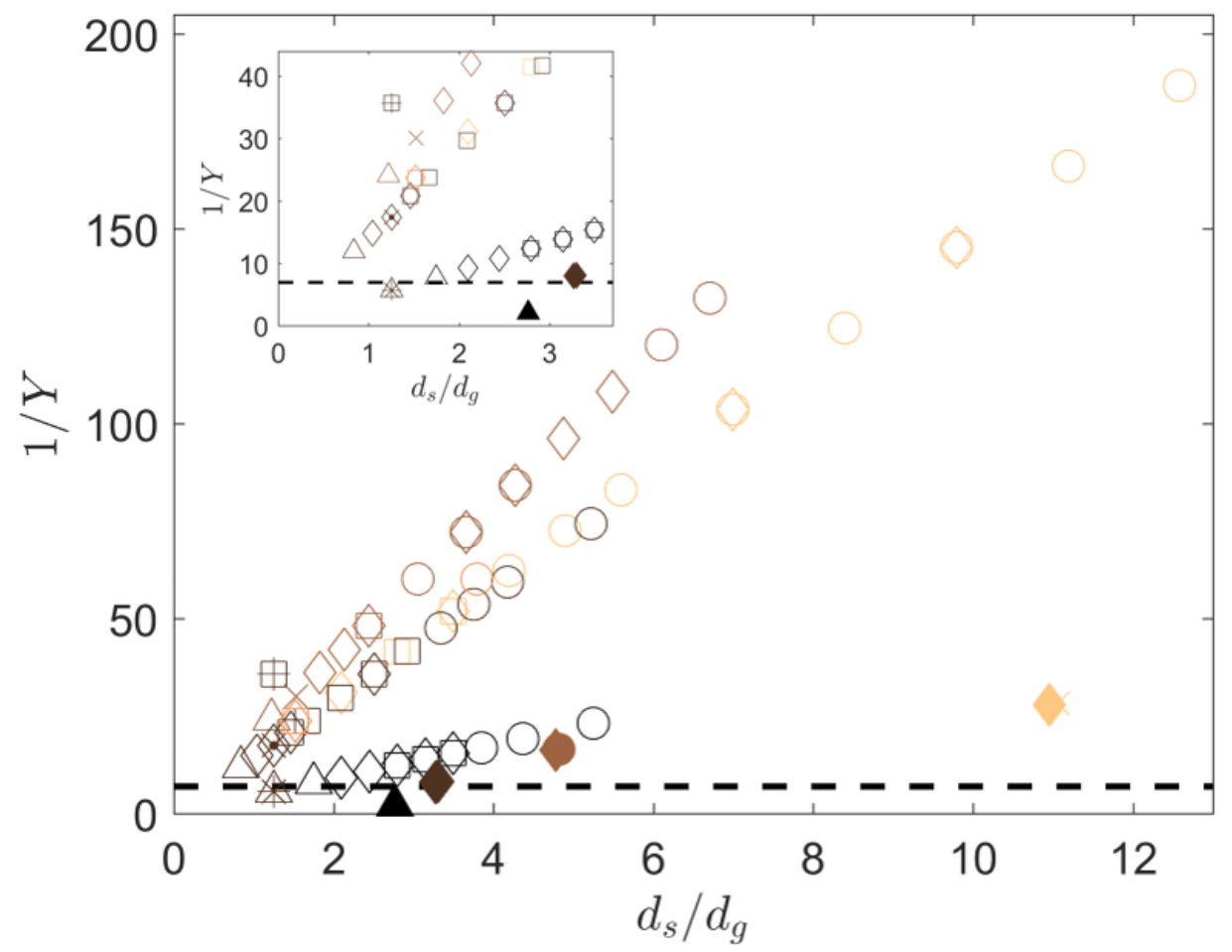

Figure 14: Range of validity of the effective rheology. Colours are for the different fluids. Symbols are those used in Fig.13. The dashed line indicates $Y_{c}^{-1}=0.145^{-1}$ [11]. For $d_{s} / d_{g}$ approaching the value of 2 , we have no motions (triangles), even for yield numbers lower than the critical one commonly used. 


\subsection{Grains rearrangement and elasticity}

The emergence of a yield stress in SAP can be related to the jamming of soft grains. Gel grains act as athermal elastic particles that interact by gliding and compressing each other. The collective behaviour of a fluid made up by these elastic grains or particles can shows elasto-viscoplastic responses [5, 44]. Therefore, one can expect that also the observed irregular motion regimes can be linked to the jamming nature of the fluid. Within this framework, going from the linear to the intermittent regime by decreasing the sphere size makes the elasticity and the yield stress seen by the sphere no longer the collective one of the bulk material, but rather the one of few gel grains. Once this occurs, to allow the continuous fall of the sphere, grains in front of it must undergo rearrangement. The mechanics of the flow appears then similar to the one of soft glassy materials. In this case the flow occurs through a sequence of elastic (reversible) deformations and local plastic (irreversible) rearrangement events which induce a long ranged relaxation of stress $[6$, 45, 46]. Grains rearrangements will be all the more common the higher the local shear stresses are (e.g. in the case of a larger sphere). Since the number of plastic events for unit of time controls fluid flow and relaxation [6], a sufficient high number of them leads the system to be more fluid. The resulting sphere motion would be in the linear regime (1); whereas irregular and intermittent regimes might be the result of a less fluid system where the local rheology seen by the sphere becomes more relevant. In this way, the observed deformation induced by the sphere falling in the linear regime is mainly the elastic interaction between the grains, as it is for flow of soft particle glasses [5]. 
Fluid elasticity have been also linked to a peculiar characteristic of the fluid flow around the sphere, namely its asymmetry. The rise of a fore-arc asymmetry and the formation of a negative wake at the sphere's tail has been detected experimentally in Carbopol [e.g. 17, 38] and associated with fluid elasticity $[13,17]$ or the emergence of thixotropy [18]. In SAP, we have observed a fore-arc asymmetry in the velocity field collected from PIV measurements. Indeed, Fig.15 shows that a negative wake in the region at the sphere back can be recognized at least for the linear motion regime (1). Although to discern the cause that breaks the fore-arc symmetry in SAP is not straightforward, its formation is more probably due to elasticity. In fact, between SAP grains there are not polymer chains that form system-spanning networks and the fluid, during a single experiment, does not undergo aging. More details about will be given in a coming work where the fluid velocity field will be properly analyzed and explained.

Moreover, a further analysis on how the properties of a single elastic grain could influence $\sigma_{Y(e f f)}$ and $K_{v(e f f)}$ might be relevant to evaluate the role that its viscoelasticity plays for the bulk rheology. However, the extreme softness of the material makes it very difficult. After several attempts to measure forces and penetration depth by using a microindenter with a minimum normal force of $20 \mathrm{mN}$, we have not been able to get any reliable measurements since slip or rupture of the grain always occurred even for such small values of normal force. 


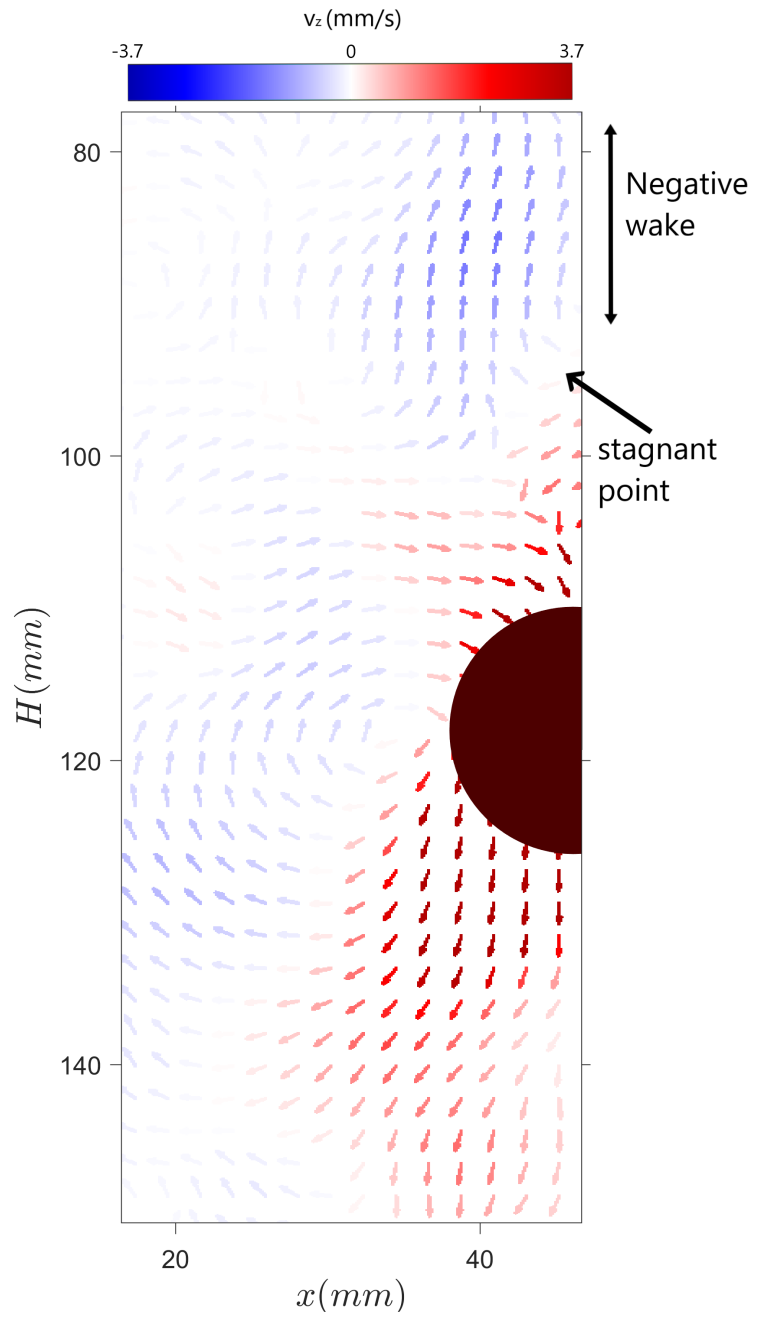

Figure 15: Fluid flow from PIV measurements. We display the vertical component of the right half part of instant velocity field $\left(v_{z}\right)$ for a sphere $\left(d_{s}=19 \mathrm{~mm}\right)$ falling in the linear regime (1) through SAP. 


\section{Conclusion}

In this work we have experimentally investigated the influence that the size of particles which build up the structure of an yield stress fluid has on the dynamics of a spherical intruder falling under gravity through it. As the typical size of the gel grains was varied between 1 and $6 \mathrm{~mm}$ and the sphere diameter was varied between 3 and $25 \mathrm{~mm}$, there exists a range where they become comparable.

We have been able to expand from three (in Carbopol) to five (in SAP) the observed regimes of motion of the sphere: (1) a linear regime where the sphere has a steady-state motion; (2) an irregular regime superimposed to a linear one where spheres never stop during their way down but their velocity varies; (3) an intermittent regime in which periods of no-motion and periods of irregular falls follow one another; (4) a logarithmic regime where the sphere velocity progressively decreases; (5) no-motion. Beside the classical steadystate motion (1) and stoppage regimes (5), typical of viscoplastic fluids, the interaction between moving objects and the fluid structure results then in two additional regimes $((2)$ and $(3))$ where the motion becomes more chaotic. The same strong interaction between the sphere and the gel grains is also observed from the sphere total travelled distance in the horizontal direction. Moreover, we have shown that the effective rheology of SAP follows the Herschel-Bulkley model with a power index $n=0.50$, making this simple setup a good rheometer for this type of fluids.

We also found that both yield stress and consistency of the gel increase with the gel grain size, suggesting that the effective rheology, due to the material heterogeneous properties, depends on the size of the particles which 
456 constitute the fluid structure. However, it is remarkable that the overall 457 sphere motion predicted by the Herschel-Bulkley model begins to "see" the ${ }_{458}$ texture only for sphere diameters less than twice the SAP grain size. 


\section{Acknowledgments}

N.R. Sgreva is supported by the Initial Training Network No 642029-ITN CREEP, an Horizon 2020 - Marie Sklodowska-Curie Action.

\section{References}

\section{References}

[1] H. A. Makse, J. Brujic, S. F. Edwards, Statistical mechanics of jammed matter, arXiv preprint cond-mat/0503081 (2005).

[2] D. Bonn, M. M. Denn, L. Berthier, T. Divoux, S. Manneville, Yield stress materials in soft condensed matter, Reviews of Modern Physics 89 (3) (2017) 035005.

[3] P. Coussot, Rheometry of pastes, suspensions, and granular materials: applications in industry and environment, John Wiley \& Sons, 2005.

[4] H. A. Barnes, A review of the slip (wall depletion) of polymer solutions, emulsions and particle suspensions in viscometers: its cause, character, and cure, Journal of Non-Newtonian Fluid Mechanics 56 (3) (1995) 221251.

[5] J. R. Seth, L. Mohan, C. Locatelli-Champagne, M. Cloitre, R. T. Bonnecaze, A micromechanical model to predict the flow of soft particle glasses, Nature materials 10 (11) (2011) 838-843.

[6] J. Goyon, A. Colin, G. Ovarlez, A. Ajdari, L. Bocquet, Spatial cooperativity in soft glassy flows, Nature 454 (7200) (2008) 84-87. 
[7] J. Goyon, A. Colin, L. Bocquet, How does a soft glassy material flow: finite size effects, non local rheology, and flow cooperativity, Soft Matter 6 (12) (2010) 2668-2678.

[8] H. de Cagny, A. Fall, M. M. Denn, D. Bonn, Local rheology of suspensions and dry granular materials, Journal of Rheology 59 (4) (2015) 957-969.

[9] D. W. De Kort, S. J. Veen, H. Van As, D. Bonn, K. P. Velikov, J. P. Van Duynhoven, Yielding and flow of cellulose microfibril dispersions in the presence of a charged polymer, Soft Matter 12 (21) (2016) 47394744 .

[10] A. Beris, J. Tsamopoulos, R. Armstrong, R. Brown, Creeping motion of a sphere through a bingham plastic, Journal of Fluid Mechanics 158 (1985) 219-244.

[11] H. Tabuteau, P. Coussot, J. R. de Bruyn, Drag force on a sphere in steady motion through a yield-stress fluid, Journal of rheology 51 (1) (2007) 125-137.

[12] N. J. Balmforth, I. A. Frigaard, G. Ovarlez, Yielding to stress: recent developments in viscoplastic fluid mechanics, Annual Review of Fluid Mechanics 46 (2014) 121-146.

[13] D. Fraggedakis, Y. Dimakopoulos, J. Tsamopoulos, Yielding the yieldstress analysis: a study focused on the effects of elasticity on the settling of a single spherical particle in simple yield-stress fluids, Soft matter 12 (24) (2016) 5378-5401. 
[14] J. Blackery, E. Mitsoulis, Creeping motion of a sphere in tubes filled with a bingham plastic material, Journal of non-newtonian fluid mechanics $70(1-2)(1997)$ 59-77.

[15] B. Deglo de Besses, A. Magnin, P. Jay, Sphere drag in a viscoplastic fluid, AIChE journal 50 (10) (2004) 2627-2629.

[16] B. T. Liu, S. J. Muller, M. M. Denn, Convergence of a regularization method for creeping flow of a bingham material about a rigid sphere, Journal of non-newtonian fluid mechanics 102 (2) (2002) 179-191.

[17] A. Putz, T. Burghelea, I. Frigaard, D. Martinez, Settling of an isolated spherical particle in a yield stress shear thinning fluid, Physics of Fluids 20 (3) (2008) 033102.

[18] M. Dinkgreve, M. Fazilati, M. Denn, D. Bonn, Carbopol: From a simple to a thixotropic yield stress fluid, Journal of Rheology 62 (3) (2018) 773780.

[19] B. Gueslin, L. Talini, Y. Peysson, Sphere settling in an aging yield stress fluid: link between the induced flows and the rheological behavior, Rheologica Acta 48 (9) (2009) 961.

[20] O. Hassager, Negative wake behind bubbles in non-newtonian liquids, Nature 279 (5712) (1979) 402.

[21] M. T. Arigo, G. H. McKinley, An experimental investigation of negative wakes behind spheres settling in a shear-thinning viscoelastic fluid, Rheologica Acta 37 (4) (1998) 307-327. 
[22] A. Mollinger, E. Cornelissen, B. Van den Brule, An unexpected phenomenon observed in particle settling: oscillating falling spheres, Journal of non-newtonian fluid mechanics 86 (3) (1999) 389-393.

[23] T. Jiang, A. Young, A. Metzner, The rheological characterization of hpg gels: Measurement of slip velocities in capillary tubes, Rheologica Acta 25 (4) (1986) 397-404.

[24] A. Jayaraman, A. Belmonte, Oscillations of a solid sphere falling through a wormlike micellar fluid, Physical Review E 67 (6) (2003) 065301.

[25] N. Kumar, S. Majumdar, A. Sood, R. Govindarajan, S. Ramaswamy, A. Sood, Oscillatory settling in wormlike-micelle solutions: bursts and a long time scale, Soft Matter 8 (16) (2012) 4310-4313.

[26] M. Fazilati, N. Maleki-Jirsaraei, S. Rouhani, D. Bonn, Quasi-periodic and irregular motion of a solid sphere falling through a thixotropic yieldstress fluid, Applied Physics Express 10 (11) (2017) 117301.

[27] P. Weidman, B. Roberts, S. Eisen, On the instability of spheres settling through a vertical pipe filled with hpg., Journal of Applied Fluid Mechanics 5 (4) (2012).

[28] S. von Kann, J. H. Snoeijer, D. Lohse, D. van der Meer, Nonmonotonic settling of a sphere in a cornstarch suspension, Physical Review E 84 (6) (2011) 060401.

[29] S. Von Kann, J. H. Snoeijer, D. Van Der Meer, Velocity oscillations and stop-go cycles: The trajectory of an object settling in a cornstarch suspension, Physical Review E 87 (4) (2013) 042301. 
[30] M. J. Zohuriaan-Mehr, K. Kabiri, Superabsorbent polymer materials: a review, Iranian polymer journal 17 (6) (2008) 451.

[31] M. Ramazani-Harandi, M. Zohuriaan-Mehr, A. Yousefi, A. ErshadLangroudi, K. Kabiri, Rheological determination of the swollen gel strength of superabsorbent polymer hydrogels, Polymer testing 25 (4) (2006) 470-474.

[32] M. R. Guilherme, F. A. Aouada, A. R. Fajardo, A. F. Martins, A. T. Paulino, M. F. Davi, A. F. Rubira, E. C. Muniz, Superabsorbent hydrogels based on polysaccharides for application in agriculture as soil conditioner and nutrient carrier: A review, European Polymer Journal 72 (2015) 365-385.

[33] R. Po, Water-absorbent polymers: a patent survey, Journal of Macromolecular Science, Part C: Polymer Reviews 34 (4) (1994) 607-662.

[34] A. T. Graham, F. L. Buchholz, Modern superabsorbent polymer technology, Wiley-vch, 1998.

[35] H. Zhang, R. S. Challa, B. Bai, X. Tang, J. Wang, Using screening test results to predict the effective viscosity of swollen superabsorbent polymer particles extrusion through an open fracture, Industrial \& Engineering Chemistry Research 49 (23) (2010) 12284-12293.

[36] L. Biehl, D. Landgrebe, Multispeca tool for multispectral-hyperspectral image data analysis, Computers \& Geosciences 28 (10) (2002) 11531159. 
[37] Blob analysis in computer vision system tm matlab (2019), https://www . mathworks.com/help/vision/ref/blobanalysis . html?s_tid=doc_ta.

[38] Y. Holenberg, O. M. Lavrenteva, U. Shavit, A. Nir, Particle tracking velocimetry and particle image velocimetry study of the slow motion of rough and smooth solid spheres in a yield-stress fluid, Physical Review E 86 (6) (2012) 066301.

[39] J. Happel, B. Howard, Low Reynolds number hydrodynamics: with special applications to particulate media, Springer Science and Business Media, 2012.

[40] T. Ferroir, H. Huynh, X. Chateau, P. Coussot, Motion of a solid object through a pasty (thixotropic) fluid, Physics of Fluids 16 (3) (2004) 594601.

[41] P. Coussot, Yield stress fluid flows: A review of experimental data, Journal of Non-Newtonian Fluid Mechanics 211 (2014) 31-49.

[42] R. W. Ansley, T. N. Smith, Motion of spherical particles in a bingham plastic, AIChE Journal 13 (6) (1967) 1193-1196.

[43] D. Atapattu, R. Chhabra, P. Uhlherr, Wall effect for spheres falling at small Reynolds number in a viscoplastic medium, Journal of nonnewtonian fluid mechanics 38 (1) (1990) 31-42.

[44] J. R. Seth, M. Cloitre, R. T. Bonnecaze, Influence of short-range forces on wall-slip in microgel pastes, Journal of Rheology 52 (5) (2008) 12411268. 
${ }_{593}^{5}$ [45] V. Mansard, A. Colin, Local and non local rheology of concentrated

594

595

596

597 particles, Soft Matter 8 (15) (2012) 4025-4043.

[46] J.-L. Barrat, A. Lemaitre, Heterogeneities in amorphous systems under shear, Dynamical heterogeneities in glasses, colloids, and granular media 150 (2011) 264. 